\title{
Distributed Self-healing Scheme for Unbalanced Electrical Distribution Systems Based on Alternating Direction Method of Multipliers
}

Shen, Feifan; López, Juan Camilo ; Wu, Qiuwei; Rider, Marcos J. ; Lu, Tianguang ; Hatziargyriou, Nikos D.

Published in:

IEEE Transactions on Power Systems

Link to article, DOI:

10.1109/TPWRS.2019.2958090

$10.1109 /$ tpwrs.2019.2958090

Publication date:

2020

Document Version

Peer reviewed version

Link back to DTU Orbit

Citation (APA):

Shen, F., López, J. C., Wu, Q., Rider, M. J., Lu, T., \& Hatziargyriou, N. D. (2020). Distributed Self-healing Scheme for Unbalanced Electrical Distribution Systems Based on Alternating Direction Method of Multipliers. IEEE Transactions on Power Systems, 35(3), 2190 - 2199. https://doi.org/10.1109/TPWRS.2019.2958090, https://doi.org/10.1109/tpwrs.2019.2958090

\section{General rights}

Copyright and moral rights for the publications made accessible in the public portal are retained by the authors and/or other copyright owners and it is a condition of accessing publications that users recognise and abide by the legal requirements associated with these rights.

- Users may download and print one copy of any publication from the public portal for the purpose of private study or research.

- You may not further distribute the material or use it for any profit-making activity or commercial gain

- You may freely distribute the URL identifying the publication in the public portal 


\title{
Distributed Self-healing Scheme for Unbalanced Electrical Distribution Systems Based on Alternating Direction Method of Multipliers
}

\author{
Feifan Shen, Juan Camilo López, Qiuwei Wu, Senior Member, IEEE, \\ Marcos J. Rider, Senior Member, IEEE, Tianguang Lu and Nikos D. Hatziargyriou, Fellow, IEEE
}

\begin{abstract}
With the advent of Smart Grids and advanced communication technologies, the self-healing scheme has become a desirable function of the operation and planning of electrical distribution systems (EDSs). In the presence of a permanent fault, an optimized self-healing scheme minimizes the unsupplied demand while maintaining the faulted section of the network isolated. The service restoration of the self-healing scheme is a combinatorial optimization problem whose computational complexity grows exponentially with the number of binary variables. To resolve this issue, a distributed optimal service restoration strategy is developed based on the alternating direction method of multipliers (ADMM). The service restoration problem is formulated as a mixed-integer second-order cone programming (MISOCP) problem. The decision variables of the problem are the status of the remote-controlled switches, load zones and load shedding at each controllable demand. Operational constraints, such as current and voltage magnitude constraints, distributed generation (DG) capacity constraints and radial topology constraints, are respected in the optimization problem. Through the ADMM, the optimization problem is distributed among the zones of the EDS, without requiring a central controller. Two test systems, an unbalanced 44-node system and the IEEE 123node system, were used to conduct case studies. Results show that the proposed method can provide optimal service restoration solutions in reasonable time without a central controller.
\end{abstract}

Index Terms-Alternating direction method of multipliers, distributed self-healing scheme, electrical distribution systems, service restoration.

\section{NOMENCLATURE}

The notation used throughout this paper is listed below. Sets and indices:

$\Omega_{\mathrm{b}} \quad$ Set of nodes

$\Omega_{\mathrm{z}}, \Omega_{\mathrm{z}}^{*} \quad$ Set of zones and set of zones without main sources

$\Phi \quad$ Set of phases $a, b, c$

This work was supported in part by the DTU-NTU double PhD project of the Smart City joint program and in part the Brazilian institution FAPESP Corresponding Author: Q. Wu (e-mail: qw@elektro.dtu.dk).

F. Shen and Q. Wu are with the Center for Electric Power and Energy, Department of Electrical Engineering, Technical University of Denmark (DTU), kgs. Lyngby, Denmark (email: fshen@elektro.dtu.dk, qw@elektro.dtu.dk).

J. C. López and M. J. Rider are with the Department of Systems and Energy, School of Electrical and Computer Engineering, University of Campinas - UNICAMP, Campinas - SP, 13083-852, Brazil (e-mail: \{jclopeza; mjrider\}@dsee.fee.unicamp.br).

T. Lu is with the School of Engineering and Applied Sciences, Harvard University, Cambridge, MA 02138, USA (e-mail: tlu@seas.harvard.edu).

N. D. Hatziargyriou is with the School of Electrical and Computer Engineering, National Technical University of Athens (NTUA), 15773 Athens, Greece (e-mail:nh@power.ece.ntua.gr).
$N_{\mathrm{p}}(i)$

$N_{\mathrm{c}}(i)$

$N_{\mathrm{p}}^{\mathrm{s}}(i)$

$N_{\mathrm{c}}^{\mathrm{s}}(i)$

$N_{\mathrm{c}}^{\mathrm{b}}(i)$

$N_{\mathrm{b}}(z)$

$i, j, z, \phi$

Variables:

$P_{i, \phi}^{j}, Q_{i, \phi}^{j}$

$I_{i, \phi}^{j, \mathrm{sqr}}$

$P_{i, \phi}^{\mathrm{G}}, Q_{i, \phi}^{\mathrm{G}}$

$V_{i, \phi}^{\mathrm{sqr}}$

$r_{i, \phi}$

$s_{i}^{j}$

$w_{i}^{j}$

$x_{z}$

$\beta_{i}^{j}, \beta_{j}^{i}$

Parameters:

$c_{i}^{\mathrm{G}}$
$c_{i}^{\mathrm{lss}, j}$

$c_{i}^{\mathrm{r}}$

$c_{i}^{\mathrm{s}, j}$

$c_{z}^{\mathrm{U}}$
$\bar{I}_{i, \phi}^{j \mathrm{sqr}}$

$\bar{P}_{i, \phi}^{\mathrm{G}}, \bar{Q}_{i, \phi}^{\mathrm{G}}$

$P_{i, \phi}^{\mathrm{D}}, Q_{i, \phi}^{\mathrm{D}}$
Set of parent nodes of node $i \in \Omega_{\mathrm{b}}$

Set of child nodes of node $i \in \Omega_{\mathrm{b}}$

Set of parent nodes of node $i$ connected by switches

Set of child nodes of node $i$ connected by switches Set of child nodes of node $i$ connected by branches

Set of nodes that belong to zone $z \in \Omega_{\mathrm{z}}$

Node $i$ and $j \in \Omega_{\mathrm{b}}$, zone $z \in \Omega_{\mathrm{z}}$ and phase $\phi \in \Phi$

Active and reactive power flow from node $i$ to $j \in N_{\mathrm{c}}(i)$, at phase $\phi$ [MW, Mvar]

Square current magnitude from node $i$ to $j \in$ $N_{\mathrm{c}}(i)$, at phase $\phi\left[\mathrm{kA}^{2}\right]$

Active and reactive generation at node $i$, at phase $\phi$ [MW, Mvar]

Square voltage magnitude at node $i$, at phase $\phi$ $\left[\mathrm{kV}^{2}\right]$

Load shedding at node $i$, at phase $\phi$ [pu]

Number of switching actions of the switch between nodes $i$ and $j \in N_{\mathrm{c}}^{\mathrm{s}}(i)$

Status of the switch between nodes $i$ and $j \in$ $N_{\mathrm{c}}^{\mathrm{s}}(i)$, where $w_{i}^{j}=0$ if the corresponding switch is open; otherwise, $w_{i}^{j}=1$ if closed

Status of zone $z$, where $x_{z}=0$ if zone $z$ is deenergized; otherwise, $x_{z}=1$ if energized

Variables associated with the switches between nodes $i$ and $j \in N_{\mathrm{c}}^{\mathrm{s}}(i)$.

Cost of generation at node $i$ [m.u./MW]

Cost of active power losses at the circuit between nodes $i$ and $j \in N_{\mathrm{c}}(i)$ [m.u./MW]

Cost of load shedding at node $i$ [m.u./MW]

Cost of a switching action of the switch between nodes $i$ and $j \in N_{\mathrm{c}}^{\mathrm{s}}(i)$ [m.u.]

Cost of de-energizing zone $z$ [m.u.]

Maximum square current magnitude of the circuit between nodes $i$ and $j \in N_{\mathrm{c}}(i)$, at phase $\phi$ [kA] Maximum active and reactive power generated at node $i$, at phase $\phi$

Active and reactive demand at node $i$, at phase $\phi$ [MW, Mvar] 
$R_{i, \phi}^{j}, X_{i, \phi}^{j}$ Resistance and reactance of the circuit between nodes $i$ and $j \in N_{\mathrm{c}}(i)$, at phase $\phi[\Omega]$

$T \quad$ Operational time of the restored system

$\bar{V}, V \quad$ Maximum and minimum voltage magnitude $[\mathrm{kV}]$

$w_{i}^{\mathrm{ini}, j} \quad$ Initial status of the switch connecting nodes $i$ and $j \in N_{\mathrm{c}}^{\mathrm{s}}(i)$

$\epsilon \quad$ ADMM convergence tolerance

$\rho \quad$ ADMM adjustable parameter

\section{INTRODUCTION}

$\mathbf{I}$ $\mathrm{N}$ electrical distribution systems (EDSs), self-healing scheme is performed by a set of equipment, algorithms and communication technologies that, after a permanent fault, establish and deploy actions to detect the fault location, isolate the faulted section of the network and restore the system with minimized unsupplied demands [1]. The selfhealing scheme must be able to provide feasible and optimized restoration solutions, with minimal human intervention, in a reasonable amount of time and with limited computational resources [2]. This is possible in smart grids with the advanced metering infrastructure (AMI) and distribution automation (DA) devices, such as remote-controlled switches, dispatchable distributed generation (DG) units and controllable loads, integrated through a telecommunication network [3].

Depending on the communication and control architecture, self-healing schemes can be classified into centralized and distributed approaches, as shown in Fig. 11. Note that without communication, neither optimality nor feasibility can be guaranteed since any decision made in one part of the EDS affects the rest of the system [4]. Most service restoration methods in the literature can be grouped into one of the two aforementioned approaches [5]. Among centralized approaches, expert systems [6], [7], meta-heuristics [8], [9], fuzzy logic [10], [11], graph theory [12], [13] and mathematical programming [2], [14]-[17] have been used to solve the service restoration problem. The main advantage of centralized methods is the ability of formulating and solving complex instances of the service restoration problem, considering multi-objective optimization [9], [18] and stochasticity [19], [20]. However, centralized methods require powerful central controllers and the computational complexity grows significantly with the increasing number of binary variables since service restoration is a combinatorial non-linear optimization problem. Moreover, centralized methods need to collect data of the overall system and may have issues with the privacy information protection.

Distributed approaches have less computational burdens because the restoration plans are obtained using multiple independent agents. The multi-agent systems (MASs) have been widely used for the operation of EDSs [21] and many MAS-based distributed service restoration schemes have been developed [22]-[25]. However, most of these formulations use simple heuristic rules that disregard the mathematical programming aspect of service restoration. Thus, the optimality of solutions cannot be guaranteed with the MAS-based approaches. An alternating direction method of multipliers $(\mathrm{ADMM})$ based distributed service restoration strategy was developed in [26] to achieve the optimal restoration plan.

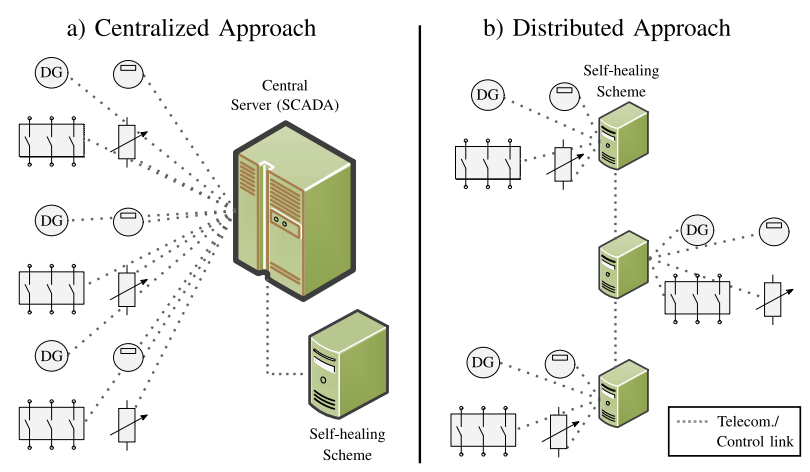

Fig. 1. Centralized vs distributed self-healing schemes.

However, network reconfiguration as an important option to facilitate service restoration is not considered in the model.

To resolve the above issues, a distributed optimal service restoration strategy for unbalanced EDSs is proposed based on the ADMM, which can obtain optimal service restoration plans in a distributed manner. Compared with the method in [26], network reconfiguration is considered in the proposed strategy, in which the optimal service restoration problem is formulated as a mixed-integer programming (MIP) model considering radial topology constraints. To decompose the MIP model and solve it in a distributed manner, a set of radial topology constraints with a decomposable structure is introduced and the binary variables are handled by projection functions in the ADMM-based algorithm. Firstly, the optimal service restoration problem is formulated as a mixedinteger non-linear programming (MINLP) model, including decomposable radiality constraints. Then, the MINLP model is transformed into a mixed-integer second-order cone programming (MISOCP) model. Finally, the MISOCP model is decomposed and solved using an ADMM-based algorithm. Through the proposed decomposition, the service restoration problem is distributed among the zones of the EDS, where each zone is a radial portion of the network separated by the remote-controlled switches. Each zone has a unique service restoration controller that exchanges information with neighbor controllers. The contributions of the paper are twofold:

1) Propose a MISOCP formulation of the optimal service restoration for unbalanced EDSs with decomposable radial topology constraints.

2) Develop an ADMM-based algorithm for distributing and solving the proposed MISOCP model, considering AC power flow, dispatchable DG units and remote-controlled loads and switches.

This paper is organized as follows: Section II presents the proposed optimal service restoration model. The proposed ADMM-based algorithm is described in Section III. Case studies are provided in Section IV, followed by conclusions.

\section{Optimal Service Restoration Model}

The optimal service restoration problem of the unbalanced EDS can be represented by a MINLP model shown in (1)(16). In the EDS, every node $i \in \Omega_{\mathrm{b}}$ belongs to a unique zone $z \in \Omega_{\mathrm{z}}$, which is an interconnected section of the network separated by remote-controlled switches. 


\section{A. Objective Function}

The objective function in (1) consists of five terms: the first term minimizes the number of de-energized zones, the second term minimizes the energy production costs, the third term minimizes the number of switching operations, the fourth term minimizes the cost of load shedding, and the last term minimizes the active power losses.

$$
\begin{aligned}
& \min \left\{\sum_{z \in \Omega_{\mathrm{z}}} c_{z}^{\mathrm{U}}\left(1-x_{z}\right)+\sum_{i \in \Omega_{\mathrm{b}}} \sum_{\phi \in \Phi} T c_{i}^{\mathrm{G}} P_{i . \phi}^{\mathrm{G}}+\right. \\
& \sum_{i \in \Omega_{\mathrm{b}}} \sum_{j \in N_{\mathrm{c}}^{\mathrm{s}}(i)} c_{i}^{\mathrm{s}, j} s_{i}^{j}+\sum_{i \in \Omega_{\mathrm{b}}} \sum_{\phi \in \Phi} T c_{i}^{\mathrm{r}} r_{i, \phi} P_{i, \phi}^{\mathrm{D}}+ \\
&\left.\sum_{i \in \Omega_{\mathrm{b}}} \sum_{j \in N_{\mathrm{c}}(i)} \sum_{\phi \in \Phi} T c_{i, \phi}^{\mathrm{lss}, j} I_{i, \phi}^{j, \mathrm{sqr}} R_{i}^{j}\right\}
\end{aligned}
$$

\section{B. Constraints}

Each constraint of the MINLP model is defined for each zone $z \in \Omega_{\mathrm{z}}$, as follows.

$$
\begin{aligned}
& P_{i, \phi}^{\mathrm{G}}+\sum_{j \in N_{\mathrm{p}}(i)} P_{j, \phi}^{i}-\sum_{j \in N_{\mathrm{c}}(i)}\left(P_{i, \phi}^{j}+I_{i, \phi}^{j, \mathrm{sqr}} R_{i, \phi}^{j}\right) \\
& =P_{i, \phi}^{\mathrm{D}}\left(x_{z}-r_{i, \phi}\right) ; \quad \forall i \in N_{\mathrm{b}}(z), \phi \in \Phi \\
& Q_{i, \phi}^{\mathrm{G}}+\sum_{j \in N_{\mathrm{p}}(i)} Q_{j, \phi}^{i}-\sum_{j \in N_{\mathrm{c}}(i)}\left(Q_{i, \phi}^{j}+I_{i, \phi}^{j, \mathrm{sqr}} X_{i, \phi}^{j}\right) \\
& =Q_{i, \phi}^{\mathrm{D}}\left(x_{z}-r_{i, \phi}\right) ; \quad \forall i \in N_{\mathrm{b}}(z), \phi \in \Phi \\
& V_{i, \phi}^{\mathrm{sqr}}-V_{j, \phi}^{\mathrm{sqr}}=2\left(P_{i, \phi}^{j} R_{i, \phi}^{j}+Q_{i, \phi}^{j} X_{i, \phi}^{j}\right)+\left(R_{i, \phi}^{j}\right)^{2} I_{i, \phi}^{j, \mathrm{sqr}}+ \\
& \left(X_{i, \phi}^{j}\right)^{2} I_{i, \phi}^{j, \mathrm{sqr}} ; \quad \forall i \in N_{\mathrm{b}}(z), j \in N_{\mathrm{c}}^{\mathrm{b}}(i), \phi \in \Phi \\
& -\bar{V}^{2}\left(1-w_{i}^{j}\right) \leq V_{i, \phi}^{\mathrm{sqr}}-V_{j, \phi}^{\mathrm{sqr}} \leq \bar{V}^{2}\left(1-w_{i}^{j}\right) \text {; } \\
& \forall i \in N_{\mathrm{b}}(z), j \in N_{\mathrm{c}}^{\mathrm{s}}(i), \phi \in \Phi \\
& 0 \leq r_{i, \phi} \leq x_{z} \\
& \forall i \in N_{\mathrm{b}}(z), \phi \in \Phi \\
& V_{j, \phi}^{\mathrm{sqr}} I_{i, \phi}^{j, \mathrm{sqr}}=\left(P_{i, \phi}^{j}\right)^{2}+\left(Q_{i, \phi}^{j}\right)^{2} ; \\
& \forall i \in N_{\mathrm{b}}(z), j \in N_{\mathrm{c}}(i), \phi \in \Phi \\
& x_{z} \underline{V}^{2} \leq V_{i, \phi}^{\mathrm{sqr}} \leq x_{z} \bar{V}^{2} ; \\
& \forall i \in N_{\mathrm{b}}(z), \phi \in \Phi \\
& \left\{\begin{array}{l}
I_{i, \phi}^{j, \mathrm{sqr}} \leq x_{z} \bar{I}_{i, \phi}^{j, \mathrm{sqr}} \\
I^{j, \mathrm{sqr}} \leq w_{i}^{j} \bar{T}_{i, \phi}^{j, \mathrm{sqr}}
\end{array}\right. \\
& \forall i \in N_{\mathrm{b}}(z), j \in N_{\mathrm{c}}^{\mathrm{b}}(i), \phi \in \Phi \\
& \forall i \in N_{\mathrm{b}}(z), j \in N_{\mathrm{c}}^{\mathrm{s}}(i), \phi \in \Phi \\
& \left\{\begin{array}{l}
0 \leq P_{i, \phi}^{\mathrm{G}} \leq \bar{P}_{i, \phi}^{\mathrm{G}} ; \\
0 \leq Q_{i, \phi}^{\mathrm{G}} \leq \bar{Q}_{i, \phi}^{\mathrm{G}} ;
\end{array}\right. \\
& \forall i \in N_{\mathrm{b}}(z), \phi \in \Phi \\
& \forall i \in N_{\mathrm{b}}(z), \phi \in \Phi \\
& \left\{s_{i}^{j} \geq w_{i}^{j}-w_{i}^{\text {ini }, j}\right. \text {; } \\
& \left\{s_{i}^{j} \geq w_{i}^{\text {ini }, j}-w_{i}^{j}\right. \text {; } \\
& x_{z}, w_{i}^{j} \in\{0,1\} \text {; } \\
& \forall i \in N_{\mathrm{b}}(z), j \in N_{\mathrm{c}}^{\mathrm{s}}(i) \\
& \forall i \in N_{\mathrm{b}}(z), j \in N_{\mathrm{c}}^{\mathrm{s}}(i) \\
& \forall i \in \Omega_{\mathrm{b}}, j \in N_{\mathrm{c}}^{\mathrm{s}}(i)
\end{aligned}
$$

Constraints (2) and (3) represent the three-phase active and reactive power balance at each node of each zone, respectively. The voltage drop of each branch at each phase is calculated in (4). Constraint (5) guarantees that if a given switch is closed (i.e., $w_{i}^{j}=1$ ), the voltage magnitudes at two ends of the switch are identical; otherwise, they can vary freely within their limits. Constraint (6) limits the percentage of load shedding at each node and each phase: if a given zone is deenergized (i.e., $x_{z}=0$ ), the amount of load shedding at all nodes in the zone is set to be zero (i.e., $r_{i, \phi}=0$ ). Current magnitudes of all branches and switches at each phase are calculated in (7). Constraint (8) limits the voltage magnitudes: if a given zone is de-energized, voltage magnitudes are forced to zero. Constraint (9) limits the current magnitudes of all branches and switches at each phase: if a given zone is deenergized, current magnitudes of branches within the zone are forced to zero. If a switch is open, the current magnitude of the switch is forced to zero. Constraint (10) limits the maximum active and reactive power generated at each node and phase. Switching operations are modeled in (11). Finally, the binary nature of $x_{z}$ and $w_{i}^{j}$ is defined in (12).

After restoration, radial topology of the energized portion of the EDS is guaranteed by power balance constraints (2)-(3) and the spanning tree constraints (13)-(16) [27]. Compared with conventional radiality constraints proposed in [28], constraints $\sqrt{13}-(16)$ are defined for each zone.

$$
\beta_{i}^{j}+\beta_{j}^{i}=w_{i}^{j} ; \quad \forall z \in \Omega_{\mathrm{z}}, i \in N_{\mathrm{b}}(z), j \in N_{\mathrm{c}}^{\mathrm{s}}(i)
$$

$$
\sum_{i \in N(z)}\left(\sum_{j \in N_{\mathrm{p}}^{\mathrm{s}}(i)} \beta_{i}^{j}+\sum_{j \in N_{\mathrm{c}}^{\mathrm{s}}(i)} \beta_{i}^{j}\right)=x_{z} ; \quad \forall z \in \Omega_{\mathrm{z}}^{*}
$$

$\beta_{i}^{j}=0$;

$$
\begin{array}{r}
\forall z \in \Omega_{\mathrm{z}} \backslash \Omega_{\mathrm{z}}^{*}, i \in N_{\mathrm{b}}(z), j \in N_{\mathrm{c}}^{\mathrm{s}}(i) \\
\forall z \in \Omega_{\mathrm{z}}, i \in N_{\mathrm{b}}(z), j \in N_{\mathrm{c}}^{\mathrm{s}}(i)
\end{array}
$$

$\beta_{i}^{j}, \beta_{j}^{i} \in[0,1]$;

\section{Formulation of the MISOCP Model}

Due to expression (7), the optimal service restoration model in (12-16) is a non-convex combinatorial problem, which is hard to solve. Thus, a relaxation technique is used to transform the MINLP problem into a MISOCP problem, which can be efficiently solved using several commercial solvers based on convex optimization techniques. A convex relaxation is applied by converting (7) into the second-order cone inequality in (17), which results in a MISOCP problem, given by (17)-(6) and (8)(17). According to [15], [29], the convex relaxation is exact if 1) the resulting energized portion of the EDS is connected and has a radial topology, 2) the objective function is linear and convex, strictly increasing with the square of current, non-decreasing with the power injection and independent of the branch power flow, 3) power injections do not reach lower bounds at the optimal solution, and 4) the problem is feasible. The convex relaxation in this paper is exact because it complies with the above mentioned conditions. It is checked that the equality (7) is satisfied for all the tests in Section IV.

$$
V_{j, \phi}^{\mathrm{sqr}} I_{i, \phi}^{j, \mathrm{sqr}} \geq\left(P_{i, \phi}^{j}\right)^{2}+\left(Q_{i, \phi}^{j}\right)^{2}
$$

As shown in the proposed MISOCP problem, each constraint is defined for each zone of the EDS, resulting in a decomposable structure that can be exploited by distributed optimization techniques, such as ADMM, as explained in the following section. 


\section{ADMM-BASEd Algorithm For the SERVICE RESTORATION PROBLEM}

ADMM has been widely used to distribute and solve convex optimization problems [30], such as the convexified optimal power flow [31], [32], optimal power-gas flow [33] and voltage control problems [34]. It takes the form of an iterative procedure, in which a group of variables are optimized first (sub-problem I), and then another group of variables are optimized in the second step (sub-problem II), exchanging information of updated primal and dual variables among sub-problems. Although ADMM is originally designed to solve convex problems, some variations of ADMM have been proposed to solve non-convex instances, such as mixedinteger linear programming (MILP) problems [35], [36] and cardinality constrained problems [37]. The practical evidence in these studies suggests that $\mathrm{ADMM}$ is also an effective method to obtain quality solutions for nonconvex problems [35].

In this section, an adaption of the ADMM algorithm is used to solve the optimal service restoration problem in a distributed manner. Firstly, the MISOCP problem is reformulated for the implementation of the ADMM algorithm. Then, the augmented Lagrangian of the problem is introduced. Finally, the ADMM-based algorithm and two sub-problems are presented.

\section{A. Reformulation of the MISOCP Model}

For brevity, phase indices $\phi$ in variables are omitted. Define set $\mathcal{K}:=\left(P_{i}^{j}, Q_{i}^{j}, \beta_{i}^{j}, V_{i}^{\mathrm{sqr}}, P_{i}^{\mathrm{G}}, r_{i}, I_{i}^{j}, s_{i}^{j}\right)$. All variables in $\mathcal{K}$ have the subscript of $i$ and are considered as exclusively affiliated variables of node $i$. In order to implement the distributed algorithm, each zone should solve its own subproblem with exclusive variables. However, as shown in (2)(5), (13) and (17), affiliated variables of node $i$, e.g., $P_{i}^{j}$ in (2), are coupled with the variables of node $j \in N_{\mathrm{p}}(i)$, e.g., $P_{j}^{i}$ in (2), which results in a coupling of adjacent zones. Therefore, a set of auxiliary variables $\left(\tilde{P}_{i}^{j}, \tilde{Q}_{i}^{j}, \tilde{\beta}_{i}^{j}, \hat{\beta}_{i}^{j}, \tilde{V}_{i}^{j, \text { sqr }}\right)$ and equality constraints shown in $(18)-(20)$ are introduced to decouple those coupled constraints.

$\left\{\begin{array}{l}P_{j}^{i}=\tilde{P}_{i}^{j}, Q_{j}^{i}=\tilde{Q}_{i}^{j} ; \forall z \in \Omega_{\mathrm{z}}, i \in N_{\mathrm{b}}(z), j \in N_{\mathrm{p}}(i) \\ P_{i}^{j}=\tilde{P}_{i}^{j}, Q_{i}^{j}=\tilde{Q}_{i}^{j} ; \forall z \in \Omega_{\mathrm{z}}, i \in N_{\mathrm{b}}(z), j \in N_{\mathrm{c}}(i)\end{array}\right.$

$\begin{cases}V_{i}^{\mathrm{sqr}}=\tilde{V}_{i}^{i, \mathrm{sqr}} ; \quad \forall z \in \Omega_{\mathrm{z}}, i \in N_{\mathrm{b}}(z) \\ V_{j}^{\mathrm{sqr}}=\tilde{V}_{i}^{j, \mathrm{sqr}} ; \quad \forall z \in \Omega_{\mathrm{z}}, i \in N_{\mathrm{b}}(z), j \in N_{\mathrm{c}}(i)\end{cases}$

$\left\{\begin{array}{l}\beta_{i}^{j}=\tilde{\beta}_{i}^{j}, \beta_{j}^{i}=\hat{\beta}_{i}^{j} ; \quad \forall z \in \Omega_{\mathrm{z}}, i \in N_{\mathrm{b}}(z), j \in N_{\mathrm{c}}^{\mathrm{s}}(i) \\ \beta_{i}^{j}=\tilde{\beta}_{i}^{j} ;\end{array}\right.$

By substituting the auxiliary variables into (2)-(5), (8) and (13)-(17), these constraints are transformed into equivalent constraints, in which variables are fully decoupled for each zone. Take (2) and (5) as examples, equivalent constraints (E2) and (E5) are formulated as below

\footnotetext{
${ }^{1}$ Due to limited space, equivalents (E3), (E4), (E8), and (E13)-(E17) are not displayed. However, analog transformations are applied to obtain these equivalent constraints.
}

$$
\begin{array}{r}
P_{i}^{\mathrm{G}}+\sum_{j \in N_{\mathrm{p}}(i)} \tilde{P}_{i}^{j}-\sum_{j \in N_{\mathrm{c}}(i)}\left(\tilde{P}_{i}^{j}+I_{i}^{j, \mathrm{sqr}} R_{i}^{j}\right)=P_{i}^{\mathrm{D}}\left(x_{z}-r_{i}\right) ; \\
\forall z \in \Omega_{\mathrm{z}}, i \in N_{\mathrm{b}}(z)(\text { E2 }) \\
-\bar{V}^{2}\left(1-w_{i}^{j}\right) \leq \tilde{V}_{i}^{i, \mathrm{sqr}}-\tilde{V}_{i}^{j, \mathrm{sqr}} \leq \bar{V}^{2}\left(1-w_{i}^{j}\right) ; \\
\forall z \in \Omega_{\mathrm{z}}, i \in N_{\mathrm{b}}(z), j \in N_{\mathrm{c}}^{\mathrm{s}}(i)(\text { E5 })
\end{array}
$$

Moreover, due to the non-convexity of the problem, dual variables may not represent the real change of the objective value when the right sides of constraints change [38]. Therefore, dual variables cannot be trusted. Here, binary variables $\left(x_{z}, w_{i}^{j}\right)$ are defined as continuous, and two additional equality constraints and auxiliary binary variables $\left(\tilde{x}_{z}, \tilde{w}_{i}^{j}\right)$ are introduced as follows:

$$
\begin{cases}x_{z}=\tilde{x}_{z} ; & \forall z \in \Omega_{\mathrm{z}} \\ w_{i}^{j}=\tilde{w}_{i}^{j} ; & \forall z \in \Omega_{\mathrm{z}}, i \in N_{\mathrm{b}}(z), j \in N_{\mathrm{c}}^{\mathrm{s}}(i) \\ \tilde{x}_{z}, \tilde{w}_{i}^{j} \in\{0,1\} ; & \forall z \in \Omega_{\mathrm{z}}, i \in \Omega_{\mathrm{d}}, j \in N_{\mathrm{c}}^{\mathrm{s}}(i)\end{cases}
$$

Finally, the MISOCP problem can be reformulated as, minimize (1): $f\left(x_{z}, P_{i}^{\mathrm{G}}, r_{i}, I_{i}^{j, \mathrm{sqr}}, s_{i}^{j}\right)$; subject to: (6), (9)-(11), (E2)-(E5), (E8), (E13)-(E17) and (18)-21).

\section{B. Augmented Lagrangian}

The augmented Lagrangian is formulated by adding equality constraints (18)-21) into the objective function of the MISOCP problem through dual variables $(\boldsymbol{\Lambda})$ defined in $(23)$, as follows:

$$
\begin{aligned}
& \min f\left(x_{z}, P_{i}^{\mathrm{G}}, r_{i}, I_{i}^{j, \mathrm{sqr}}, s_{i}^{j}\right)+\frac{1}{2} \rho\left\{\sum _ { z \in \Omega _ { \mathrm { z } } } \sum _ { i \in N _ { \mathrm { b } } ( z ) } \left[\sum_{j \in N_{\mathrm{p}}(i)}\right.\right. \\
& \left(\left\|\tilde{P}_{i}^{j}-P_{j}^{i}+\mu_{i}^{j}\right\|^{2}-\left\|\mu_{i}^{j}\right\|^{2}\right)+\sum_{j \in N_{\mathrm{c}}(i)}\left(\left\|\tilde{P}_{i}^{j}-P_{i}^{j}+\chi_{i}^{j}\right\|^{2}\right. \\
& \left.-\left\|\chi_{i}^{j}\right\|^{2}\right)+\sum_{j \in N_{\mathrm{p}}(i)}\left(\left\|\tilde{Q}_{i}^{j}-Q_{j}^{i}+\kappa_{i}^{j}\right\|^{2}-\left\|\kappa_{i}^{j}\right\|^{2}\right)+\sum_{j \in N_{\mathrm{c}}(i)} \\
& \left(\left\|\tilde{Q}_{i}^{j}-Q_{i}^{j}+\lambda_{i}^{j}\right\|^{2}-\left\|\lambda_{i}^{j}\right\|^{2}\right)+\left(\left\|\tilde{V}_{i}^{i, \mathrm{sqr}}-V_{i}^{\mathrm{sqr}}+\pi_{i}\right\|^{2}-\right. \\
& \left.\left\|\pi_{i}\right\|^{2}\right)+\sum_{j \in N_{\mathrm{c}}(i)}\left(\left\|\tilde{V}_{i}^{j, \mathrm{sqr}}-V_{j}^{\mathrm{sqr}}+\varsigma_{i}^{j}\right\|^{2}-\left\|\varsigma_{i}^{j}\right\|^{2}\right)+ \\
& \sum_{j \in N_{\mathrm{c}}^{\mathrm{s}}(i)}\left(\left\|\tilde{\beta}_{i}^{j}-\beta_{i}^{j}+\tau_{i}^{j}\right\|^{2}-\left\|\tau_{i}^{j}\right\|^{2}\right)+\sum_{j \in N_{\mathrm{p}}^{\mathrm{s}}(i)}\left(\| \tilde{\beta}_{i}^{j}-\beta_{i}^{j}\right. \\
& \left.\left.+\psi_{i}^{j}\left\|^{2}-\right\| \psi_{i}^{j} \|^{2}\right)+\sum_{j \in N_{\mathrm{c}}^{\mathrm{s}}(i)}\left(\left\|\hat{\beta}_{i}^{j}-\beta_{j}^{i}+\phi_{i}^{j}\right\|^{2}-\left\|\phi_{i}^{j}\right\|^{2}\right)\right] \\
& +\sum_{z \in \Omega_{\mathrm{z}}}\left[\left(\left\|x_{z}-\tilde{x}_{z}+\varpi_{z}\right\|^{2}-\left\|\varpi_{z}\right\|^{2}\right)+\sum_{i \in N_{\mathrm{b}}(z)} \sum_{j \in N_{\mathrm{c}}^{\mathrm{s}}}\left(\| w_{i}^{j}\right.\right. \\
& \left.\left.\left.-\tilde{w}_{i}^{j}+\zeta_{i}^{j}\left\|^{2}-\right\| \zeta_{i}^{j} \|^{2}\right)\right]\right\} \quad(22
\end{aligned}
$$

Subject to: 66, 9)-(11) and (E2)-(E5), (E8), (E13)-(E17).

Note that binary variables are only included in the objective function (22) of the augmented lagrangian and all constraints of the lagrangian function can be decomposed into each zone. Here, the augmented lagrangian is optimized over two groups of primal variables $(\mathbf{X}, \mathbf{Y})$ and one group of dual variables $(\boldsymbol{\Lambda})$, 
as defined in (23). For ease of presentation, (22) is expressed in a compact form by $g(\mathbf{X}, \mathbf{Y}, \mathbf{\Lambda})$.

$$
\left\{\begin{array}{l}
\mathbf{X}=\left[P_{i}^{\mathrm{G}}, r_{i}, I_{i}^{j, \mathrm{sqr}}, s_{i}^{j}, \tilde{P}_{i}^{j}, \tilde{Q}_{i}^{j}, \tilde{V}_{i}^{j}, \tilde{\beta}_{i}^{j}, \hat{\beta}_{i}^{j}, x_{z}, w_{i}^{j}\right] \\
\mathbf{Y}=\left[P_{i}^{j}, Q_{i}^{j}, V_{i}, \beta_{i}^{j}, \tilde{x}_{z}, \tilde{w}_{i}^{j}\right] \\
\boldsymbol{\Lambda}=\left[\mu_{i}^{j}, \chi_{i}^{j}, \kappa_{i}^{j}, \lambda_{i}^{j}, \pi_{i}, \varsigma_{i}^{j}, \tau_{i}^{j}, \psi_{i}^{j}, \phi_{i}^{j}, \varpi_{z}, \zeta_{i}^{j}\right]
\end{array}\right.
$$

\section{ADMM-based Algorithm}

In the ADMM-based algorithm, optimization over primal variables is separated into two sub-problems (sub-problems I and II). Primal variables $(\mathbf{X}, \mathbf{Y})$ and dual variables $(\boldsymbol{\Lambda})$ are updated in an iterative process, as follows:

1) Sub-problem I: At the $k$-th iteration, sub-problem I is given by 24, subject to constraints of the augmented Lagrangian, in which primal variables $\mathbf{X}^{k}$ are optimized, and primal variables $\mathbf{Y}$ and dual variables $\boldsymbol{\Lambda}$ are considered as parameters (i.e., $\mathbf{Y}^{*, k-1}$ and $\boldsymbol{\Lambda}^{*, k-1}$ ) obtained in the $(k-1)$ th iteration.

$$
\text { Sub-problem I : } g\left(\mathbf{X}^{k}, \mathbf{Y}^{*, k-1}, \boldsymbol{\Lambda}^{*, k-1}\right)
$$

Subject to: (6), (9)-(11) and (E2)-(E5), (E8), (E13)-(E17).

Note that sub-problem $\mathrm{I}$ is formulated as a second-order cone programming (SOCP) problem and can be decomposed into each zone and solved in a distributed manner.

2) Sub-problem II: After solving sub-problem I, subproblem II in 25) is solved at $k$-th iteration to optimize the primal variables $\mathbf{Y}^{k}$ with the obtained $\mathbf{X}^{*, k}$ and $\boldsymbol{\Lambda}^{*, k-1}$.

$$
\text { Sub-problem II : } g\left(\mathbf{X}^{*, k}, \mathbf{Y}^{k}, \boldsymbol{\Lambda}^{*, k-1}\right)
$$

Note that sub-problem II is a non-constrained mixed integer quadratic programming (MIQP) problem due to the binary nature of $\tilde{x}_{z}$ and $\tilde{w}_{i}^{j}$. Instead of solving a MIQP problem, the two terms in 25 associated with $\tilde{x}_{z}$ and $\tilde{w}_{i}^{j}$ are excluded and the problem is solved as a quadratic programming $(\mathrm{QP})$ problem. Then, the binary variables are obtained using the following projection function:

$$
\begin{cases}\tilde{x}_{z}^{k}=\prod_{x}\left(x_{z}^{*, k}+\varpi_{z}^{*, k-1}\right) ; & \forall z \in \Omega_{\mathrm{z}} \\ \tilde{w}_{i}^{j, k}=\prod_{w}\left(w_{i}^{j, *, k}+\zeta_{i}^{j, *, k-1}\right) ; & \forall z \in \Omega_{\mathrm{z}}, \\ & i \in N_{\mathrm{b}}(z), j \in N_{\mathrm{p}}^{\mathrm{s}}(i)\end{cases}
$$

Here, $\prod$ denotes the projection onto $x$ and $w$. Since $\tilde{x}_{z}$ and $\tilde{w}_{i}^{j}$ are binary variables, $\prod$ rounds each entry to its nearest binary value. Since the sub-problem II is a non-constrained problem and the binary variables are not coupled with other decision variables, using the projection function and solving the MIQP sub-problem II directly are equivalent in terms of obtaining solutions of binary variables. It is worth noting that, without constraints, sub-problem II can be easily calculated for each zone in a distributed manner.

3) Dual variables update: Among zones, the exchanged primal variables associated with the dual variables are $\mathbf{X}^{\text {ex }}$ defined in (27) and $\mathbf{Y}$. Once the exchanged variables are updated using the two sub-problems at the $k$-th iteration, dual variables can be updated using (28).

$$
\begin{aligned}
& \mathbf{X}^{\mathrm{ex}}=\left[\tilde{P}_{i}^{j}, \tilde{Q}_{i}^{j}, \tilde{V}_{i}^{j}, \tilde{\beta}_{i}^{j}, \hat{\beta}_{i}^{j}, x_{z}, w_{i}^{j}\right] ; \\
& \mathbf{\Lambda}^{*, k}=\boldsymbol{\Lambda}^{*, k-1}+\left(\mathbf{X}^{\mathrm{ex}, *, \mathrm{k}}-\mathbf{Y}^{*, k}\right) ;
\end{aligned}
$$

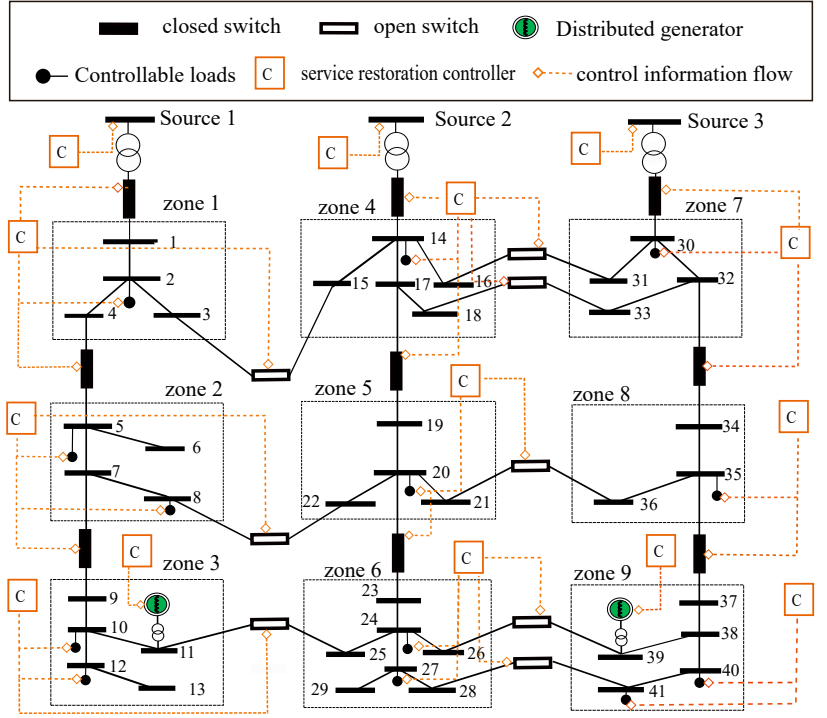

Fig. 2. Initial topology of the unbalanced 44-node test system.

4) Stopping criterion: Primal variables and dual variables are updated sequentially until the aggregated residual between exchanged variables is lower than a specified tolerance, as shown in 29].

$\left\|\mathbf{X}^{\mathrm{ex} *}-\mathbf{Y}^{*}\right\| \leq \epsilon$;

\section{Tests And Results}

To validate the efficiency of the proposed distributed selfhealing scheme, two case studies were conducted with an unbalanced 44-node test system and an IEEE 123-node system. The proposed ADMM-based algorithm was implemented in GAMS [39], and solutions were obtained using CPLEX [40]. In the ADMM-based algorithm, the weight coefficients are determined based on the priority of each objective [16]. Restoring power for outage zones and loads is the main goal of service restoration and has the top priority. The minimization of the number of switching operations is to reduce restoration time and has the second priority. The power losses reduction has the least priority. Therefore, the cost of de-energizing a zone $c_{z}^{\mathrm{U}}$ is set as 50 m.u. (monetary units), the cost of load shedding $c_{i}^{\mathrm{r}}$ is set as $10 \mathrm{~m} . \mathrm{u} . / \mathrm{MWh}$, the cost of a switching action $c_{i}^{\mathrm{s}, j}$ is set as 5 m.u., and the cost of energy losses $c_{i}^{\text {lss }, j}$ is set as 0.1 m.u./MWh. The generation cost coefficients of the main sources and DG units are approximately based on their generation costs. The cost coefficient $c_{i}^{\mathrm{G}}$ is set as 0.2 m.u./MWh for each main source and set as 0.1 m.u./MWh for each DG unit. The tolerance $\epsilon$ is $1 \times 10^{-3}$ and adjustable parameter $\rho$ is 350 in Case 1, and 200 in Case 2. The operational time of the restored system $T$ is 1 hour. The lower limit of the voltage magnitude is set as $0.95 \mathrm{pu}$ in both cases.

\section{A. Case 1: unbalanced 44-node test system}

In this case, an unbalanced 44-node test system shown in Fig. 2 is used to verify the effectiveness of the proposed ADMM-based algorithm. The system consists of three separated feeders with nine zones and two DG units connected 
TABLE I

SUMMARY OF RESTORATION RESULTS

\begin{tabular}{|c|c|c|c|c|c|c|c|c|c|c|c|}
\hline \multirow[b]{2}{*}{ cases } & \multirow[b]{2}{*}{ scenarios } & \multirow[b]{2}{*}{$\begin{array}{l}\text { faulty } \\
\text { zone }\end{array}$} & \multirow{2}{*}{\multicolumn{2}{|c|}{ switching operations }} & \multirow{2}{*}{$\begin{array}{l}\text { power } \\
\text { losses } \\
{[\mathrm{kW}]}\end{array}$} & \multirow[b]{2}{*}{$V^{\min }[\mathrm{pu}]$} & \multirow[b]{2}{*}{$I^{\max }[\mathrm{A}]$} & \multicolumn{2}{|c|}{ load shedding } & \multicolumn{2}{|c|}{ loads served } \\
\hline & & & & & & & & $\begin{array}{c}P \\
{[\mathrm{~kW}]}\end{array}$ & $\begin{array}{c}Q \\
{[\mathrm{kvar}]}\end{array}$ & $\begin{array}{c}P \\
{[\mathrm{~kW}]}\end{array}$ & $\begin{array}{c}Q \\
{[\mathrm{kvar}]}\end{array}$ \\
\hline \multirow{4}{*}{ Case 1} & no. 1 & zone 1 & r1-1, 4-5 & $8-22$ & 53.31 & $V_{13, a}=0.965^{* *}$ & $I_{\mathrm{r} 2, a}^{14}=92.4^{* * * *}$ & I & I & 4800 & 960 \\
\hline & no. 2 & zone 1 & $\mathrm{r} 1-1,4-5$ & $11-25$ & 77.46 & $V_{8, a}=0.950$ & $I_{\mathrm{r} 2, a}^{14}=99.9$ & 48.27 & 9.65 & 4751.73 & 950.35 \\
\hline & no. 3 & $\begin{array}{l}\text { source } \\
\text { node } 1\end{array}$ & r1-1 & $3-15$ & 46.34 & $V_{41, a}=0.966$ & $I_{\mathrm{r} 2, a}^{14}=104.6$ & l & l & 4800 & 960 \\
\hline & no. 4 & zones 2,5 & $\begin{array}{c}4-5,7-9, \\
17-19,20-23\end{array}$ & $\begin{array}{l}11-25 \\
26-39 \\
\end{array}$ & 58.05 & $V_{13, a}=0.950$ & $I_{\mathrm{r} 3, a}^{30}=112.7$ & 61.48 & 12.30 & 4738.52 & 947.70 \\
\hline \multirow{4}{*}{ Case 2} & no. 1 & zone 2 & $13-18,18-135$ & $39-66$ & 32.98 & $V_{51, a}=0.971$ & $I_{\mathrm{r} 2, a}^{300}=118.4$ & I & I & 3490 & 1920 \\
\hline & no. 2 & zone 2 & $13-18,18-135$ & $151-300$ & 26.32 & $V_{94, a}=0.977$ & $I_{\mathrm{r} 2, a}^{300}=143.3$ & l & I & 3490 & 1920 \\
\hline & no. 3 & zone 1 & $\begin{array}{c}13-18,13-152 \\
18-135\end{array}$ & $\begin{array}{c}151-300, \\
39-66\end{array}$ & 23.58 & $V_{52, a}=0.975$ & $I_{\mathrm{r} 2, a}^{300}=193.0$ & l & l & 3490 & 1920 \\
\hline & no. 4 & zone 1 & $13-18,13-152$ & $\begin{array}{c}151-300, \\
39-66\end{array}$ & 26.82 & $V_{52, a}=0.962$ & $I_{\mathrm{r} 2, a}^{300}=212.0$ & 62.43 & 31.22 & 3427.57 & 1888.78 \\
\hline
\end{tabular}

${ }^{*}$ r1-1: the switch between source node 1 and node 1.

${ }^{* *} V_{13, a}=0.965$ : the minimal voltage magnitude is 0.965 pu at node 13 at phase $a$.

${ }^{* * *} I_{\mathrm{r} 2, a}^{14}=92.4$ : the maximum current magnitude is $92.4 \mathrm{~A}$ between source node 2 and node 14 at phase $a$.

at zone 3 (DG 1) and zone 9 (DG 2), respectively. Open switches are represented as white-colored boxes, and closed switches are represented as black-colored boxes. Detailed data of loads and branches are given in [14]. DG units operate with unity power factors and their capacities for each phase are $100 \mathrm{~kW}$. Four scenarios were conducted in this case: In scenarios 1 and 2, a fault occurs in the load zone adjacent to the source node. The operation of both DG units is considered in scenario 1, whereas the operation of DG 1 is disregarded in scenario 2. In scenarios 3 and 4, the operation of both DG units is considered. In scenario 3, a fault occurs at the source node or upstream to the source node. In scenario 4, multiple faults in middle load zones are considered. The restoration results of the four scenarios of Case 1 are summarized in Table I

1) Scenario 1: In this scenario, the operation of all DG units is considered. It is assumed that a permanent fault occurs in zone 1. After executing the proposed ADMMbased algorithm, the restored system isolates the faulty zone by opening the switches between source 1 and node 1 and between nodes 4 and 5 . Then, the switch between nodes 8 and 22 is closed to supply zones 2 and 3 . The restored test system has $53.32 \mathrm{~kW}$ of active power losses and the minimum voltage magnitude is $0.965 \mathrm{pu}$ at node 13 at phase $a$. Current magnitudes are within their operational limits and both DG units use their maximum power capacities because they have lower energy production costs than the main sources.

2) Scenario 2: Once again, assume the same fault occurs in zone 1 while the operation of DG 1 is disregarded. After executing the proposed ADMM-based algorithm, zones 2 and 3 are supplied by closing the switch between nodes 11 and 25 instead of the switch between nodes 8 and 22. Since DG 1 is disconnected, more energy must be transmitted from the main sources to supply zone 3 . Due to the limited capacity of the switch between nodes 8 and 22, closing this switch results in a larger amount of load shedding and a higher total cost than closing the switch between nodes 11 and 25 . The restored test system sheds $24.147 \mathrm{~kW}$ and $4.829 \mathrm{kVar}$ loads at node 5 at phase $a$, and $24.120 \mathrm{~kW}$ and $4.824 \mathrm{kVar}$ loads at node 8 at phase $a$ to ensure that all operational constraints are not violated. The minimum voltage magnitude is $0.950 \mathrm{pu}$ at node 6 at phase $a$.

3) Scenario 3: In this scenario, suppose that a fault occurs at source node 1 or upstream to source node 1 . In such a case, the utility power from source node 1 is not available. After executing the proposed ADMM-based algorithm, the source node 1 is isolated by opening the switch between source node 1 and node 1 . Then, the switch between nodes 3 and 15 is closed to supply outage zones 2 and 3 . The restored system has $46.52 \mathrm{~kW}$ of active power losses. All operational constraints are respected and two DG units operate with the maximum capacities.

4) Scenario 4: In this scenario, multiple faults are simulated. Suppose that two faults occur in zones 2 and 5, respectively. After executing the proposed ADMM-based algorithm, the switches between nodes 4 and 5 and between nodes 7 and 9 are opened to isolate the faulty zone 2 . The switches between nodes 17 and 19 and between nodes 20 and 23 are opened to isolate the faulty zone 5 . Then, the switches between nodes 11 and 25 and between nodes 26 and 39 are closed to supply outages zones 3 and 6 . To ensure that voltage magnitude constraints are not violated, the system sheds $61.48 \mathrm{~kW}$ and $12.30 \mathrm{kVar}$ loads at node 12 at phase $a$. The minimum voltage magnitude is $0.950 \mathrm{pu}$ at node 13 at phase $a$. In addition, both DG units use their maximum power capacities.

\section{B. Case 2: IEEE 123-node test system}

In this case, the effectiveness of the proposed ADMMbased algorithm under switch maintenance and loss of communication is validated on the modified IEEE 123-node test system in Fig. 3. The system is unbalanced and consists of two feeders and six DG units that operate with unity power factors. Detailed load and branch data can be found in [41]. The restoration results of the four scenarios of Case 2 are summarized in Table I. 


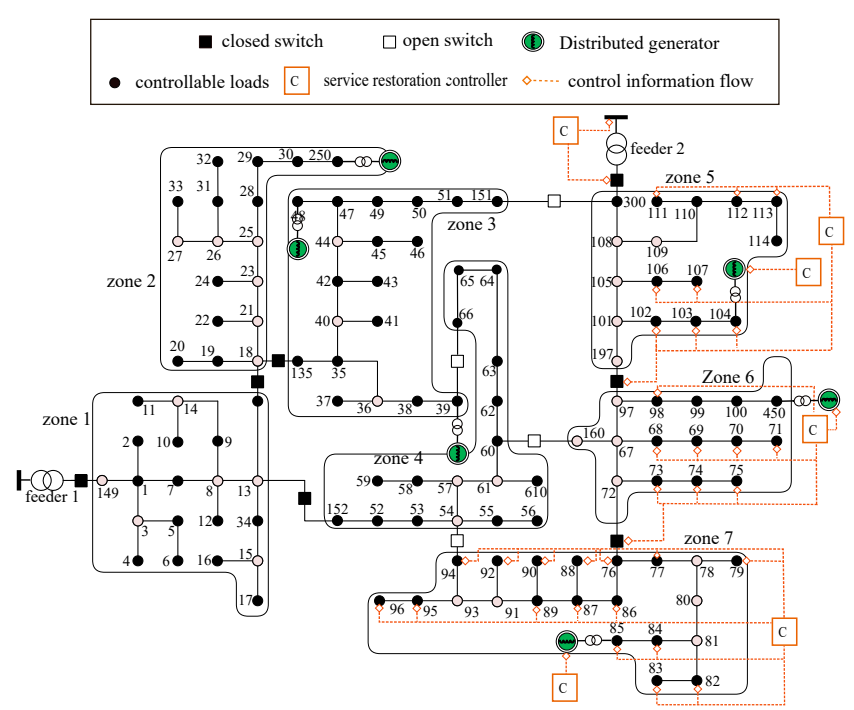

Fig. 3. Initial topology of the modified IEEE 123-node test system.

1) Scenario 1: In this scenario, a fault occurs in zone 2 and the proposed ADMM-based algorithm is implemented to restore the system. The switches between nodes 13 and 18 and between nodes 18 and 135 are opened to isolate the faulty zone. Then, the switch between nodes 39 and 66 is closed to supply zone 3 . The restored system has $32.98 \mathrm{~kW}$ of active power losses and the minimum voltage is $0.971 \mathrm{pu}$ at node 51 at phase $a$. All operation constraints are respected in this scenario. The DG unit at node 250 is disconnected and other DG units operate with their maximum power capacities.

2) Scenario 2: switch maintenance: In this scenario, the same fault in zone 2 is simulated while the switch between nodes 39 and 66 is under maintenance. In such a case, the switch between nodes 151 and 300 is closed to supply zone 3 . The restored test has $26.32 \mathrm{~kW}$ of active power losses and the minimum voltage magnitude is 0.977 pu at node 94 , phase $a$. All operational constraints are respected.

3) Scenario 3: communication loss of the zone downstream to the fault: In some situations, such as failure of communication devices, the loss of communication could happen. If all communication within a zone is lost, the self-healing scheme should guarantee that the zone without communication does not participate in the restoration process and remains its initial status prior to service restoration. It is assumed that a fault occurs in zone 1 and its downstream zone 2 loses communication. Thus, zone 2 should remain de-energized and its controller does not participate in the restoration process. After performing the proposed ADMM-based algorithm, zones 3 and 4 are supplied by closing switches between nodes 151 and 300 and between nodes 39 and 66. All operational constraints are not violated.

4) Scenario 4: communication loss of the zone not downstream to the fault: In this scenario, assume the same fault in zone 1 and zone 7 loses communication. In such a case, zone 7 should remain energized and its controller does not participate in the restoration process. All variables associated with the zone, e.g., power flow at the switch between nodes 72 and 76 , are set as the values obtained in the normal operation. After executing the ADMM-based algorithm, zones 2, 3 and
TABLE II

NUMBERS OF ITERATIONS IN BOTH CASES

\begin{tabular}{ccccc}
\hline & scenario 1 & scenario 2 & scenario 3 & scenario 4 \\
\hline case 1 & 791 & 735 & 581 & 749 \\
\hline case 2 & 691 & 558 & 493 & 662 \\
\hline \hline
\end{tabular}

4 are supplied and the restored system sheds loads at nodes, e.g., sheds $18.378 \mathrm{~kW}$ and $9.189 \mathrm{kVar}$ loads at load 70 at phase $a$, to ensure that variables associated with zone 7 , e.g., voltage and current magnitudes, remain unchanged.

\section{Performance of the Proposed ADMM-based Algorithm}

1) Convergence processes: The numbers of iterations in both cases are listed in Table II Take Case 1 as an example, the convergence processes of the aggregated residuals in four scenarios are shown in Fig. 4. It can be seen that the algorithm converges to the specified tolerance after a reasonable number of iterations. As shown in Fig. 5, the binary variables associated with the status of switches between nodes 8 and 22 (switch a) and between nodes 11 and 25 (switch b) in scenarios 1 and 2, converge before 20 iterations, which demonstrates the effectiveness of the projection function to deal with binary variables.

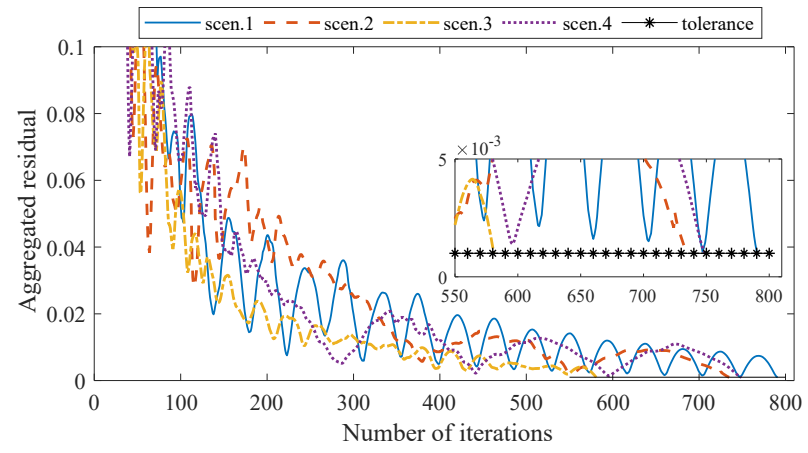

Fig. 4. Convergence of the aggregated residuals in Case 1.

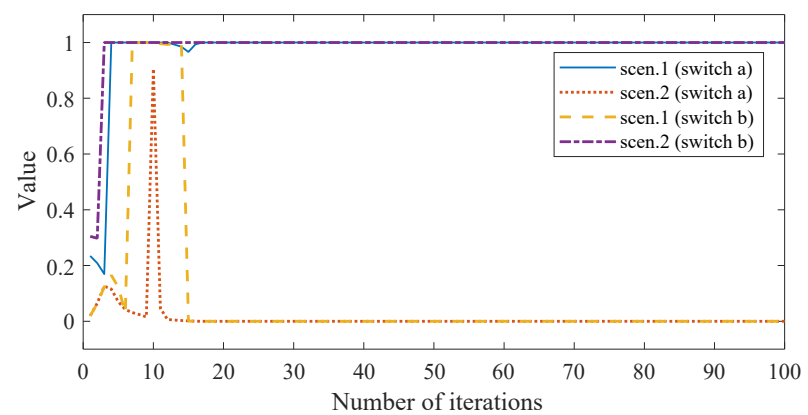

Fig. 5. Convergence of binary variables associated with switches between nodes 8 and 22 and between nodes 11 and 25 in scenarios 1 and 2, Case 1 .

2) Sensitivity analysis: Case 1 is used to study the impacts of the adjustable parameter $\rho$ and the tolerance $\epsilon$ on the convergence and the accuracy of the proposed ADMM-based algorithm. Convergence rates of Case 1 with different values of $\rho$ are shown in Table III Note that four scenarios have different numbers of iterations with the same $\rho$. Clearly, improper $\rho$ may cause slow convergence, e.g., scenarios 2 and 4 with $\rho$ of 50. Therefore, it is important to choose an appropriate value for 
TABLE III

NUMBERS OF ITERATIONS WITH DIFFERENT $\rho$ IN CASE 1

\begin{tabular}{c|ccccc}
\hline \hline$\rho$ & scenario 1 & scenario 2 & scenario 3 & scenario 4 & average \\
\hline 50 & 1332 & 2385 & 879 & 2440 & 1759.00 \\
\hline 100 & 995 & 1089 & 813 & 1051 & 987.00 \\
\hline 200 & 885 & 824 & 787 & 774 & 817.50 \\
\hline 300 & 735 & 750 & 760 & 910 & 788.75 \\
\hline $\mathbf{3 5 0}$ & 791 & $\mathbf{7 3 5}$ & 581 & $\mathbf{7 4 9}$ & $\mathbf{7 1 4 . 0 0}$ \\
\hline 400 & 787 & 1090 & 582 & 746 & 801.25 \\
\hline 500 & 744 & 1100 & 695 & 914 & 863.25 \\
\hline 600 & $\mathbf{6 5 4}$ & 1290 & $\mathbf{5 4 3}$ & 1086 & 893.25 \\
\hline \hline
\end{tabular}

TABLE IV

ACCURACY AND NUMBERS OF ITERATIONS WITH DIFFERENT CONVERGence TOlerances $(\epsilon)$ FOR CASE 1

\begin{tabular}{c|c|ccc}
\hline \hline \multicolumn{2}{c|}{$\epsilon$} & $1 \times 10^{-2}$ & $1 \times 10^{-3}$ & $1 \times 10^{-4}$ \\
\hline \multirow{4}{*}{$\begin{array}{c}\text { relative } \\
\text { error }\end{array}$} & scenario 1 & $1.41 \times 10^{-1}$ & $5.45 \times 10^{-4}$ & $4.40 \times 10^{-4}$ \\
\cline { 2 - 5 } & scenario 2 & $2.19 \times 10^{-1}$ & $7.67 \times 10^{-3}$ & $1.60 \times 10^{-3}$ \\
\cline { 2 - 5 } & scenario 3 & $4.91 \times 10^{-3}$ & $6.21 \times 10^{-4}$ & $3.44 \times 10^{-4}$ \\
\cline { 2 - 5 } & scenario 4 & 5.71 & $1.26 \times 10^{-2}$ & $3.10 \times 10^{-3}$ \\
\cline { 2 - 5 } & average & 1.52 & $5.40 \times 10^{-3}$ & $1.40 \times 10^{-3}$ \\
\hline \multirow{4}{*}{$\begin{array}{c}\text { number } \\
\text { of iterations }\end{array}$} & scenario 1 & 221 & 791 & 1620 \\
\cline { 2 - 5 } & scenario 2 & 368 & 735 & 1989 \\
\cline { 2 - 5 } & scenario 3 & 324 & 581 & 1176 \\
\cline { 2 - 5 } & scenario 4 & 269 & 749 & 3509 \\
\cline { 2 - 5 } & average & 295.5 & 714.0 & 2073.5 \\
\hline \hline
\end{tabular}

$\rho$. In Case $1, \rho$ is set as 350 because it has the least average number of iterations for all scenarios.

The impact of the convergence tolerance $\epsilon$ on the accuracy of solutions and the number of iterations are shown in Table IV] The accuracy of solutions obtained with the proposed ADMM-based algorithm is evaluated by the relative error (RE) using (30), where $\mathbf{s}$ is the vector of nodal power injections obtained with the proposed ADMM-based algorithm, and $\mathbf{s}^{*}$ is the vector of optimal nodal power injections obtained with the centralized algorithm. The centralized solution $\mathbf{s}^{*}$ is obtained by solving the formulated MISOCP problem in Section III using the CPLEX solver with a zero optimality gap. As shown in Table IV] the accuracy of solutions is improved as the convergence tolerance decreases. On the other hand, the algorithm requires more iterations to converge. Compared with the solution using $\epsilon=1 \times 10^{-2}$, the average RE obtained with $\epsilon=1 \times 10^{-3}$ decreases by 281 times and the average number of iterations increases by 2.41 times. Although the average $\mathrm{RE}$ of solutions can be further reduced by 3.9 times when $\epsilon=1 \times 10^{-4}$, the average number of iterations still increases by 2.9 times. Therefore, the algorithm with $\epsilon=1 \times 10^{-3}$ has a good trade-off between the accuracy and the number of iterations.

$$
\mathrm{RE}=\left\|\mathbf{s}-\mathbf{s}^{*}\right\| /\left\|\mathbf{s}^{*}\right\|
$$

\section{Comparisons Between the Centralized Method and the Proposed Distributed Method}

1) Value of the Objective Function: The RE of the value of the objective function obtained with the distributed method is shown in Table $\mathrm{V}$. It can be seen that the maximum $\mathrm{RE}$ is lower than $1.7 \times 10^{-4}$. Moreover, as shown in Table IV, the $\mathrm{RE}$ of solutions obtained with the distributed method is lower
TABLE V

RElative ERrors (REs) OF THE OBJective FunCtions IN Both CASES

\begin{tabular}{ccc}
\hline \hline & case 1 & case 2 \\
\hline scenario 1 & $1.10 \times 10^{-6}$ & $3.80 \times 10^{-6}$ \\
\hline scenario 2 & $5.75 \times 10^{-6}$ & $2.49 \times 10^{-6}$ \\
\hline scenario 3 & $3.80 \times 10^{-7}$ & $1.03 \times 10^{-6}$ \\
\hline scenario 4 & $2.84 \times 10^{-5}$ & $1.69 \times 10^{-4}$ \\
\hline \hline
\end{tabular}

TABLE VI

COMPARISON OF THE NUMBERS OF VARIABLES OF OPTIMIZATION Problems in Both Cases, Scenario 1

\begin{tabular}{c|c|c|c}
\hline \hline \multicolumn{2}{c|}{ Number of variables } & Centralized method & $\begin{array}{c}\text { Distributed method } \\
\text { (each controller) }\end{array}$ \\
\hline \multirow{2}{*}{ Case 1} & Continuous & 1046 & 134 \\
& Integer & 62 & 0 \\
\hline \multirow{2}{*}{ Case 2} & Continuous & 3666 & 443 \\
& Integer & 39 & 0 \\
\hline \hline
\end{tabular}

than $1.3 \times 10^{-2}$. Therefore, the proposed distributed method can reproduce the solutions obtained with centralized methods.

2) Computation Performance: As for the optimization problem, the centralized method solves a MISOCP problem whereas the distributed method solves smaller scale QP problems and applies projection functions. Table VI summarizes the numbers of variables of optimization problems in the two methods for both cases, scenario 1. Compared with the centralized method, the average numbers of continuous variables of each controller in each calculation are reduced by $87.19 \%$ in Case 1 , and by $87.92 \%$ in Case 2 . Moreover, there are no integer variables involved in the optimization of the distributed method. Thus, the controller in the distributed method solves smaller scale optimization problems.

The computation time of the distributed algorithm in each iteration is the duration of the most time-consuming controller in the iteration. The average computation time per iteration in each scenario in both cases is shown in Table VII Note that the average computation time per iteration in each scenario in Case 2 is larger than the one in Case 1 because each controller has more affiliated nodes in Case 2. The total computation time of the distributed algorithm and the centralized algorithm is summarized in Table VIII] It can be seen that the proposed distributed method can obtain solutions within 15 seconds in both cases, which complies with requirements of self-healing systems [42], [43]. Although the distributed method needs more computation time than the centralized method, it does not need a powerful central controller and it can provide privacy information protection, e.g., a DG unit is an independent entity participating in service restoration without posing its privacy information to other agents.

\section{CONCLUSION}

In this study, the service restoration problem of the selfhealing scheme is formulated as a MINLP model, including radiality constraints with a decomposable structure. Then, the MINLP model is transformed into a MISOCP model, which is decomposed and solved using an ADMM-based algorithm that handles binary decision variables by projection functions. With 
TABLE VII

Average Computation Time Per Iteration in Both CASES

\begin{tabular}{c|c|c}
\hline \hline second [s] & Case 1 & Case 2 \\
\hline scenario 1 & 0.0105 & 0.0211 \\
\hline scenario 2 & 0.0106 & 0.0178 \\
\hline scenario 3 & 0.0112 & 0.0166 \\
\hline scenario 4 & 0.0135 & 0.0166 \\
\hline
\end{tabular}

TABLE VIII

Computation Time of Centralized vs Distributed Methods

\begin{tabular}{c|cc|cc}
\hline \hline \multirow{2}{*}{ second [s] } & \multicolumn{2}{|c|}{ Case 1 } & \multicolumn{2}{c}{ Case 2 } \\
\cline { 2 - 5 } & Centralized & Distributed & Centralized & Distributed \\
\hline scenario 1 & 1.52 & 8.34 & 2.34 & 14.56 \\
\hline scenario 2 & 0.87 & 7.81 & 1.79 & 9.95 \\
\hline scenario 3 & 1.00 & 6.53 & 2.52 & 8.18 \\
\hline scenario 4 & 0.935 & 10.08 & 2.95 & 11.02 \\
\hline \hline
\end{tabular}

the proposed decomposition, the service restoration problem is distributed among the zones of the EDS, where each zone has a self-healing controller that exchanges information with neighbor controllers. An unbalanced 44-node system and the IEEE 123-node system were used to validate the effectiveness of the proposed distributed self-healing scheme. For all cases, the proposed distributed method isolates the faulty zones and minimizes the unsupplied demand. Moreover, the proposed distributed method can reproduce the optimal solutions obtained with the centralized method and can ensure privacy information protection.

\section{REFERENCES}

[1] J. R. Aguero, "Applying self-healing schemes to modern power distribution systems," in 2012 IEEE Power and Energy Society General Meeting, Sand Diego, CA, Jul. 2012, pp. 1-4.

[2] Z. Wang and J. Wang, "Self-healing resilient distribution systems based on sectionalization into microgrids," IEEE Trans. Power Syst., vol. 30, no. 6, pp. 3139-3149, Nov. 2015.

[3] P. Gopakumar, B. Mallikajuna, M. J. B. Reddy, and D. K. Mohanta, "Remote monitoring system for real time detection and classification of transmission line faults in a power grid using pmu measurements," Protection and Control of Modern Power Systems, vol. 3, no. 3, pp. 159-168, Dec. 2018.

[4] K. E. Antoniadou-Plytaria, I. N. Kouveliotis-Lysikatos, P. S. Georgilakis, and N. D. Hatziargyriou, "Distributed and decentralized voltage control of smart distribution networks: Models, methods, and future research," IEEE Trans. Smart Grid, vol. 8, no. 6, pp. 2999-3008, Nov. 2017.

[5] F. Shen, Q. Wu, S. Huang, J. C. López, C. Li, and B. Zhou, "Review of service restoration methods distribution networks," in 2018 IEEE PES Innovative Smart Grid Technologies Conference Europe, Sarajevo, BH, Oct. 2018, pp. 1-6.

[6] M. Tsai, "Development of an object-oriented service restoration expert system with load variations," IEEE Trans. Power Syst., vol. 23, no. 1, pp. 219-225, Feb. 2008.

[7] S. Srivastava and K. L. Butler-Burry, "Expert-system method for automatic reconfiguration for restoration of shipboard power systems," IEE Proceedings - Generation, Transmission and Distribution, vol. 153, no. 3, pp. 253-260, May 2006.

[8] S. Toune, H. Fudo, T. Genji, Y. Fukuyama, and Y. Nakanishi, "Comparative study of modern heuristic algorithms to service restoration in distribution systems," IEEE Trans. Power Del., vol. 17, no. 1, pp. 173181, Jan. 2002.

[9] L. T. Marques, A. C. B. Delbem, and J. B. A. London, "Service restoration with prioritization of customers and switches and determination of switching sequence," IEEE Trans. Smart Grid, vol. 9, no. 3, pp. 23592370, May 2018.

[10] Q. Zhou, D. Shirmohammadi, and W. E. Liu, "Distribution feeder reconfiguration for service restoration and load balancing," IEEE Trans. Power Syst., vol. 12, no. 2, pp. 724-729, May 1997.
[11] D. S. Popovic and Z. N. Popovic, "A risk management procedure for supply restoration in distribution networks," IEEE Trans. Power Syst., vol. 19, no. 1, pp. 221-228, Feb. 2004.

[12] J. Li, X. Ma, C. Liu, and K. P. Schneider, "Distribution system restoration with microgrids using spanning tree search," IEEE Trans. Power Syst., vol. 29, no. 6, pp. 3021-3029, Nov. 2014.

[13] E. Drayer, N. Kechagia, J. Hegemann, M. Braun, M. Gabel, and R. Caire, "Distributed self-healing for distribution grids with evolving search space," IEEE Trans. Power Del., vol. 33, no. 4, pp. 1755-1764, Aug. 2018.

[14] P. L. Cavalcante, J. F. F. J. C. López, M. J. Rider, A. V. Garcia, M. R. R. Malveira, L. L. Martins, and L. c. M. Direito, "Centralized self-healing scheme for electrical distribution systems," IEEE Trans. Smart Grid, vol. 7, no. 1, pp. 145-155, Jan. 2016.

[15] R. Romero, J. F. Franco, F. B. Leão, M. J. Rider, and E. S. de Souza, “A new mathematical model for the restoration problem in balance radial distribution system," IEEE Trans. Power Sys., vol. 31, no. 2, pp. 1259 1267, Mar. 2016

[16] J. C. López, J. F. Franco, M. J. Rider, and R. Romero, "Optimal restoration/maintenance switching sequence of unbalanced three-phase distribution systems," IEEE Trans. Smart Grid, vol. 9, no. 6, pp. 60586068, Nov. 2018

[17] B. Chen, C. Chen, J. Wang, and K. L. Butler-Purry, "Sequential service restoration for unbalanced distribution systems and microgrids," IEEE Trans. Power Syst., vol. 33, no. 2, pp. 1507-1520, Mar. 2018.

[18] Y. Kumar, B. Das, and J. Sharma, "Multiobjective, multiconstraint service restoration of electric power distribution system with priority customers," IEEE Trans. Power Del., vol. 23, no. 1, pp. 261-270, Jan. 2008.

[19] K. Chen, W. Wu, B. Zhang, and H. Sun, "Robust restoration decisionmaking model for distribution networks based on information gap decision theory," IEEE Trans. Smart Grid, vol. 6, no. 2, pp. 587-597, Mar. 2015.

[20] X. Chen, W. Wu, and B. Zhang, "Robust restoration method for active distribution networks," IEEE Trans. Power Syst., vol. 31, no. 5, pp. 4005-4015, Sep. 2016.

[21] I. Kouveliotis-Lysikatos, D. Koukoula, A. Dimeas, N. Hatziargyriou, and S. Makrynikas, "Overview of decentralized distribution system operation techniques," in CIRED Workshop, Helsinki, Finland, Jun. 2016.

[22] J. M. Solanki, S. Khushalani, and N. N. Schulz, "A multi-agent solution to distribution systems restoration," IEEE Trans. Power Syst., vol. 22, no. 3, pp. 1026-1034, Aug. 2007.

[23] A. Zidan and E. F. El-Saadany, "A cooperative multiagent framework for self-healing mechanisms in distribution systems," IEEE Trans. Smart Grid, vol. 3, no. 3, pp. 1525-1539, Sep. 2012.

[24] A. Elmitwally, M. Elsaid, M. Elgamal, and Z. Chen, "A fuzzy-multiagent service restoration scheme for distribution system with distributed generation," IEEE Trans. Sustain. Energy, vol. 6, no. 3, pp. 810-821, Jul. 2015.

[25] A. A. Hafez, W. A. Omran, and Y. G. Hegazy, "A decentralized technique for autonomous service restoration in active radial distribution networks," IEEE Trans. Smart Grid, vol. 9, no. 3, pp. 1911-1919, May 2018.

[26] R. R. Nejad and W. Sun, "Distributed load restoration in unbalanced active distribution systems," IEEE Trans. Smart Grid, in press.

[27] R. A. Jabr, R. Singh, and B. C. Pal, "Minimum loss network reconfiguration using mixed-integer convex programming," IEEE Trans. Power Syst., vol. 27, no. 2, pp. 1106-1115, May 2012.

[28] M. Lavorato, J. F. Franco, M. J. Rider, and R. Romero, "Imposing radiality constraints in distribution system optimization problems," IEEE Trans. Power Syst., vol. 27, no. 1, pp. 172-180, Feb. 2012.

[29] S. H. Low, "Convex relaxation of optimal power flow - Part II: Exactness," IEEE Trans. Control Netw. Syst., vol. 1, no. 2, pp. 177189, Jun. 2014.

[30] S. Boyd, N. Parikh, E. Chu, B. Peleato, and J. Eckstein, "Distributed optimization and statistical learning via the alternating direction method of multipliers," Foundations and Trends in Machine Learning, vol. 3, no. 1, pp. 1-122, Jan. 2010.

[31] Q. Peng and S. H. Low, "Distributed optimal power flow algorithm for radial networks, I: Balanced single phase case," IEEE Trans. Smart Grid, vol. 9, no. 1, pp. 111-121, Jan. 2018.

[32] Y. Wang, L. Wu, and S. Wang, "A fully-decentralized consensus-based admm approach for dc-opf with demand response," IEEE Trans. Smart Grid, vol. 8, no. 6, pp. 2637-2647, Nov. 2017.

[33] C. Wang, W. Wei, J. Wang, L. Bai, Y. Liang, and T. Bi, "Convex optimization based distributed optimal gas-power flow calculation," IEEE Trans. Sustain. Energy, vol. 9, no. 3, pp. 1145-1156, Jul. 2018. 
[34] S. Huang, Q. Wu, Y. Guo, X. Chen, B. Zhou, and C. Li, "Distributed voltage control based on ADMM for large-scale wind farm cluster connected to VSC-HVDC," IEEE Trans. sustain. Energy, in press.

[35] R. Takapoui, N. Moehle, S. Boyd, and A. Bemporad, "A simple effective heuristic for embedded mixed-integer quadratic programming," in Proc. American Control Conf., Boston, Massachusetts, 2016, pp. 5619-5625.

[36] A. Alborz and M. C. Rotkowitz, "Improving ADMM-based optimization of mixed integer objectives," in Annual Conf. on Information Sciences and Systems, Baltimore, MD, USA, May 2017.

[37] S. Diamond, R. Takapoui, and S. Boyd, "A general system for heuristic solution of convex problems over nonconvex sets," Optimization Methods and Sofware, vol. 33, no. 1, pp. 165-193, Apr. 2017.

[38] D. P. Bertsekas, Nonlinear Programming, 2nd ed. Athena Scientific, 1995.

[39] General Algebraic Modeling System (GAMS), GAMS Platform. [Online]. Available: :http://www.gams.com/

[40] IBM ILOG CPLEX optimization studio CPLEX user's manual.

[41] IEEE Power and Energy Society (PES). (2013) Distribution test feeders: 123-bus feeder. [Online]. Available: http://ewh.ieee.org/soc/pes/dsacom/ testfeeders/

[42] D. Bernardon, L. Canha, A. Abaide, V. Garcia, and M. Sperandio, "Smart grid concepts applied to self-healing in distribution system," in 2014 49th International Universities Power Engineering Conference (UPEC), Cluj-Napoca, Romania, Sep. 2014, pp. 1-6.

[43] Y. Lin and etc., "Towards a real-time fault identification and self-healing system in the distribution network," in IEEE Industrial and Commercial Power Systems Europe (IDCPS Europe), Milan, Italy, Jun. 2017, pp. $1-6$. 